\title{
Genetic, Structural, and Phenotypic Properties of MS2 Coliphage with Resistance to $\mathrm{ClO}_{2}$ Disinfection
}

Qingxia Zhong, ${ }^{\dagger}$ Anna Carratalà, ${ }^{\dagger}$ Sergey Nazarov, ${ }^{\dagger, \S}$ Ricardo Cesar Guerrero-Ferreira, ${ }^{\ddagger}, \|$ Laura Piccinini, ${ }^{\dagger}$ Virginie Bachmann, ${ }^{\dagger}$ Petr G. Leiman, ${ }^{,}, 1$ and Tamar Kohn*, ${ }^{\dagger}$

${ }^{\dagger}$ Laboratory of Environmental Chemistry, School of Architecture, Civil and Environmental Engineering, and ${ }^{\ddagger}$ Laboratory of Structural Biology and Biophysics, Institute of Physics, School of Basic Sciences, École Polytechnique Fédérale de Lausanne (EPFL), CH-1015 Lausanne, Switzerland

\section{Supporting Information}

ABSTRACT: Common water disinfectants like chlorine have been reported to select for resistant viruses, yet little attention has been devoted to characterizing disinfection resistance. Here, we investigated the resistance of MS2 coliphage to inactivation by chlorine dioxide $\left(\mathrm{ClO}_{2}\right) \cdot \mathrm{ClO}_{2}$ inactivates MS2 by degrading its structural proteins, thereby disrupting the ability of MS2 to attach to and infect its host. $\mathrm{ClO}_{2}$-resistant virus populations emerged not only after repeated cycles of $\mathrm{ClO}_{2}$ disinfection followed by regrowth but also after dilutionregrowth cycles in the absence of $\mathrm{ClO}_{2}$. The resistant populations exhibited several fixed mutations which caused the substitution of $\mathrm{ClO}_{2}$-labile by $\mathrm{ClO}_{2}$-stable amino acids. On
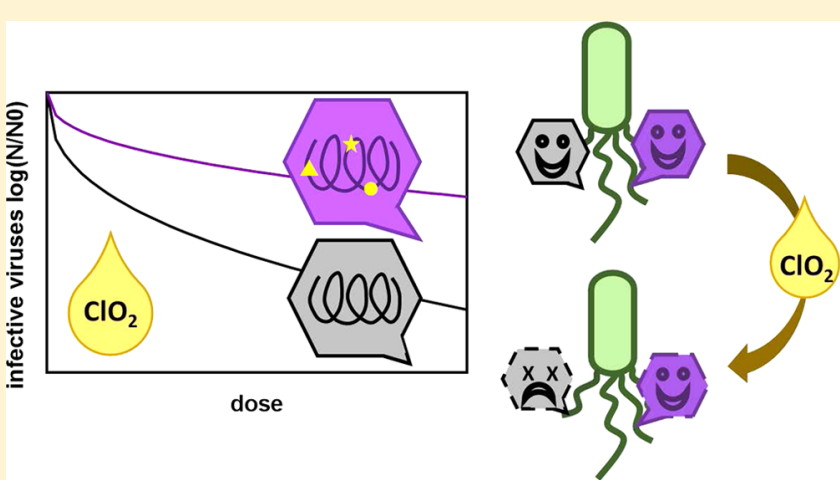
a phenotypic level, these mutations resulted in a more stable host binding during inactivation compared to the wild-type, thus resulting in a greater ability to maintain infectivity. This conclusion was supported by cryo-electron microscopy reconstruction of the virus particle, which demonstrated that most structural modification occurred in the putative A protein, an important binding factor. Resistance was specific to the inactivation mechanism of $\mathrm{ClO}_{2}$ and did not result in significant cross-resistance to genome-damaging disinfectants. Overall, our data indicate that resistant viruses may emerge even in the absence of $\mathrm{ClO}_{2}$ pressure but that they can be inactivated by other common disinfectants.

\section{INTRODUCTION}

Chemical oxidants such as free chlorine (FC) or chlorine dioxide $\left(\mathrm{ClO}_{2}\right)$ are widely used to control a number of waterborne pathogens in wastewater and drinking water. However, viruses remain a concern as they are generally more resistant toward disinfectants than traditional bacterial indicators such as Escherichia coli and Enterococci. ${ }^{1,2}$ In addition, they have very low infectious doses, ${ }^{3}$ which implies that disinfection must be very effective in order to reduce the risks associated with virally contaminated water.

Compared to other organisms, RNA viruses have high population numbers and high mutation rates that increase the genetic diversity within a population and allow them to rapidly evolve and adapt to environmental stress. ${ }^{4-8}$ It is well-known that resistant viruses readily emerge upon continuous administration of antiviral drugs and, eventually, even among naive patients that received no clinical treatment. ${ }^{9,10}$ A lesserknown fact is that viruses can also evolve resistance to common water disinfectants. For example, previous studies have demonstrated that poliovirus and F116 bacteriophage developed resistance to chlorine upon repeated exposure to the disinfectant under laboratory conditions. ${ }^{11,12}$ Similarly, it was shown that poliovirus isolated from treated drinking water with a chlorine residual exhibited greater resistance toward free chlorine as compared to laboratory-adapted strains. ${ }^{13}$ These observations suggest that virus resistance to disinfection has a genetic basis that may be influenced by evolutionary mechanisms.

To date, the adaptation of virus populations to chemical disinfection has not been characterized. This is in part due to the lack of understanding of the specific mechanisms driving virus inactivation upon exposure to disinfectants. Hence, it is difficult to unravel the molecular basis underpinning virus adaptation to disinfection. Such information, however, is important to avoid potential detrimental outcomes of disinfection, such as the selection for viruses that cannot be treated by commonly applied methods. Furthermore, a mechanistic understanding of the resistance mechanisms is important to design alternative treatment strategies for resistant viruses.

Received: August 17, 2016

Revised: September 29, 2016

Accepted: October 6, 2016

Published: October 6, 2016 
In this study we investigated the emergence of resistance of MS2 bacteriophage to disinfection by chlorine dioxide $\left(\mathrm{ClO}_{2}\right)$, and we characterized the underlying mechanisms conferring MS2 resistance to the disinfectant. We chose to work with MS2 because it is a common surrogate for enteric viruses, and because its mechanisms of inactivation by $\mathrm{ClO}_{2}$ have previously been studied. ${ }^{14}$ Specifically, $\mathrm{ClO}_{2}$ inactivates MS2 by selectively damaging susceptible regions on the structural viral proteins, while the genome remains intact. This results in the inability of MS2 to bind to its host cell and hence in its inactivation. ${ }^{14} \mathrm{We}$ therefore hypothesize that viruses with resistance to $\mathrm{ClO}_{2}$ exhibit mutations in their structural proteins that lead to an enhanced stability toward $\mathrm{ClO}_{2}$ or to alternative binding mechanisms that are less affected by the oxidant. To test this hypothesis, we conducted an experimental evolution assay to obtain $\mathrm{ClO}_{2}$-resistant MS2 populations. We then identified the mutations fixed in the resistant populations and characterized their effects with respect to the mutants' morphology, their ability to bind to the host, and their aggregation behavior. Finally, we assessed the resistant mutants' replicative fitness and their susceptibility to other inactivating agents, to obtain information regarding the propensity of the disinfectionresistant virus to proliferate in the environment.

\section{EXPERIMENTAL SECTION}

Briefly, this work is divided into four sets of experiments: first, we conducted experimental evolution studies in order to obtain MS2 population with evolved resistance to $\mathrm{ClO}_{2}$. Second, we identified the mutations fixed in $\mathrm{ClO}_{2}$-resistant MS2 populations by genome sequencing. Third, we characterized some phenotypic traits of a subset of $\mathrm{ClO}_{2}$-resistant populations (host binding, morphology, and tendency to aggregate). Lastly, we investigated the attributes related to the proliferation of resistant MS2, namely the replicative fitness, and the cross-resistance of $\mathrm{ClO}_{2}$-resistant populations against other means of inactivation (FC, $\mathrm{UV}_{254}$, and heat).

MS2 Culturing and Enumeration. Bacteriophage MS2 (DSMZ 13767) and its Escherichia coli host (DSMZ 5695) were obtained from the German Collection of Microorganisms and Cell Cultures (DSMZ, Braunschweig, Germany). Propagation and purification of all MS2 populations were conducted as described previously. ${ }^{15}$ Infectivity was assessed by enumeration of plaque forming units ( $\mathrm{pfu}$ ) using the double agar layer method. ${ }^{16}$ The stock solutions had a concentration of $10^{12} \mathrm{pfu} /$ $\mathrm{mL}$ and were stored in the refrigerator at $4{ }^{\circ} \mathrm{C}$ in phosphatebuffered saline (PBS; $5 \mathrm{mM} \mathrm{Na} \mathrm{HPO}_{4}$ (99\%, Acros), $10 \mathrm{mM}$ $\mathrm{NaCl}$ (99.5\%, Acros), pH 7.4).

Chlorine Dioxide Production. A stock solution of $\mathrm{ClO}_{2}$ was produced by mixing $100 \mathrm{~mL}$ of $4 \% \mathrm{~K}_{2} \mathrm{~S}_{2} \mathrm{O}_{8}$ (99\%, Acros) with $100 \mathrm{~mL}$ of $2 \% \mathrm{NaClO}_{2}$ (Sigma-Aldrich) and was stored at $4{ }^{\circ} \mathrm{C}$. The resulting $\mathrm{ClO}_{2}$ concentration $(250-1000 \mathrm{mg} / \mathrm{L})$ was determined spectrophotometrically (UV-2550 UV-vis spectrophotometer, Shimadzu) from the absorbance at $358 \mathrm{~nm}$ $\left(\varepsilon_{358 \mathrm{~nm}}=1200 \mathrm{M}^{-1} \mathrm{~cm}^{-1}\right) .{ }^{17}$ The stock solution was diluted with PBS to obtain $\mathrm{ClO}_{2}$ working solutions with a concentration of $0.5-4 \mathrm{mg} / \mathrm{L}$. Ultrapure water $(>18 \mathrm{M} \Omega$ $\mathrm{cm}^{-1}$ ) was used for all aqueous solutions.

Experimental Evolution Assays. In order to obtain resistant MS2 populations, we conducted experimental evolution experiments by subjecting a wild-type (WT) MS2 population to repeated cycles of $\mathrm{ClO}_{2}$ exposure (Figure S1, SI). Each cycle consisted of treating a virus population $\left(10^{9}-10^{10}\right.$ $\mathrm{pfu} / \mathrm{mL}$ ) with $\mathrm{ClO}_{2}$ such that up to 6 logs of inactivation were achieved. Following inactivation, the solution with the remaining infective viruses was concentrated using a $100 \mathrm{kDa}$ Microcon centrifugal filter (Millipore, Billerica, MA) and was washed five times with PBS. Finally $50 \mu \mathrm{L}$ of solution were collected and spiked into $12 \mathrm{~mL}$ of an E. coli culture in exponential growth phase at an approximate multiplicity of infection (MOI) of $10^{-5}$ (approximately $10^{3} \mathrm{pfu}$ viruses per $10^{8}$ cfu bacteria). Once regrown, the resulting virus populations were purified as detailed previously ${ }^{15}$ and then re-exposed to $\mathrm{ClO}_{2}$. Five replicate experimental evolution assays were conducted of $>20$ disinfection-regrowth cycles each. The five resulting populations are named E01-E05 hereafter, where E stands for "exposed to $\mathrm{ClO}_{2}$ ". The $\mathrm{ClO}_{2}$ concentration used was $0.5 \mathrm{mg} / \mathrm{L}$ at the beginning of the experimental evolution and was incrementally increased to $4 \mathrm{mg} / \mathrm{L}$ in the last cycles.

To test the role of $\mathrm{ClO}_{2}$ as a selection pressure in the experiments, five control evolution assays were performed in which MS2 populations were only subjected to repeated regrowth but not $\mathrm{ClO}_{2}$ exposure. The resulting populations are henceforth named NE01-NE05, where NE stands for "nonexposed populations". Specifically, after each regrowth step, the control populations were diluted, and approximately $10^{2}-10^{3}$ pfu were passed to the next cycle. These cycles were repeated 15 (NE01) or 22 times (NE02-05), at which point resistance had emerged.

Kinetic Inactivation Experiments with $\mathrm{ClO}_{2}$. The emergence of resistance was monitored by comparing the inactivation kinetics of the evolved populations with that of the wild-type after every 5 cycles. Inactivation experiments were carried out in triplicate at room temperature $\left(22^{\circ} \mathrm{C}\right)$ under constant stirring. Specifically, $10 \mathrm{~mL}$ beakers were rinsed with 5 $\mathrm{mL}$ of $\mathrm{ClO}_{2}$ working solution before each experiment to eliminate any $\mathrm{ClO}_{2}$ demand. Then $2 \mathrm{~mL}$ of $\mathrm{ClO}_{2}$ working solution was added to the beaker and was spiked with MS2 stock solution to obtain a starting concentration of approximately $10^{8} \mathrm{pfu} / \mathrm{mL}$. The exact initial virus titer in each experiment was determined by injecting the same volume of MS2 stock solution to a reactor containing $2 \mathrm{~mL}$ of $\mathrm{ClO}_{2}$-free PBS. Initial $\mathrm{ClO}_{2}$ concentrations ranged from 0.5 to $2 \mathrm{mg} / \mathrm{L}$. To compensate for $\mathrm{ClO}_{2}$ decay and evaporation during the experiment, a concentrated $\mathrm{ClO}_{2}$ solution $(16 \mathrm{mg} / \mathrm{L})$ was continuously added to the beaker at a flow rate of $10-25 \mu \mathrm{L} /$ min using a peristaltic pump (KdScientific). Prior to the start of each experiment it was ensured that this setup was able to maintain a constant desired $\mathrm{ClO}_{2}$ concentration. Samples (minimum $10 \mu \mathrm{L}$ ) were taken periodically over the course of 1 to 4 min and were immediately mixed with PBS containing $10 \%$ $\mathrm{w} / \mathrm{v}$ sodium thiosulfate ( $98 \%$, Sigma-Aldrich) to quench the residual disinfectant. Control samples with sodium thiosulfate added to $\mathrm{ClO}_{2}$-free virus solution confirmed that sodium thiosulfate did not cause virus inactivation.

Inactivation rate constants for $\mathrm{ClO}_{2}$ were determined by fitting the experimental data to the Hom model. ${ }^{18}$ If the disinfectant concentration is constant during inactivation the Hom model can be expressed as

$$
\ln \frac{N}{N_{0}}=-k_{\mathrm{ClO}_{2}} C^{n} t^{m}
$$

where $N_{0}$ is the initial infective virus concentration and $N$ is infective virus concentration at time $t ; k_{\mathrm{ClO}_{2}}$ is the Hom rate constant $\left[\left(\mathrm{mg} \mathrm{L}^{-1} \mathrm{~min}^{m-1}\right)^{-1}\right] ; C$ is the $\mathrm{ClO}_{2}$ concentration $(\mathrm{mg} / \mathrm{L}$; kept constant during the experiments); $m$ is a model 
parameter that describes the deviation from first-order; and $n$ is the coefficient of dilution that attributes the relative importance of time and concentration in the inactivation process. To determine the model coefficients $k_{\mathrm{ClO}_{2}}, m$, and $n$, Excel solver and GraphPad Prism were employed to minimize the sum of squares of the difference between the observed and modeled data.

Sanger Sequencing. Single nucleotide polymorphisms (SNP) potentially associated with $\mathrm{ClO}_{2}$-resistant MS2 populations were identified by Sanger sequencing as detailed in the Supporting Information.

Host Binding Assays. Binding assays were conducted to test the hypothesis that the host binding mechanism of the resistant mutant was less impacted by $\mathrm{ClO}_{2}$ than that of the wild-type. Binding of MS2 to their E. coli host was determined in triplicate and was quantified as described previously. ${ }^{19}$ Briefly, the E. coli host was grown to exponential growth phase before being inoculated with MS2 and incubated on ice for 90 min. The samples were then centrifuged, and the bacterial pellet was washed with Tris buffer to remove any free viruses and was then resuspended. The viral RNA was extracted, and quantitative RT-PCR (qRT-PCR) was performed to quantify the number of attached viruses. The $\mathrm{qRT}$-PCR reaction was performed as described previously, ${ }^{15}$ using a One Step SYBER PrimeScript RT-PCR Kit (Takara) and a Rotorgene 3000 quantitative PCR platform (Corbett Life Science).

Aggregation. Virus aggregation was assessed by dynamic light scattering and zeta potential measurements. Experimental details are provided in the Supporting Information.

Cryo-Electron Microscopy (Cryo-EM). Cryo-EM was used to reconstruct the structure of the wild-type and mutant viruses, at a 10.5 and $9.5 \AA$ resolution, respectively. The mutant population that most readily yielded good cryo-EM images (NE01) was chosen for this purpose. The relevant experimental and technical details are given in the Supporting Information. Cryo-EM maps of the WT MS2 bacteriophage and its NE01 mutant have been deposited to the Electron Microscopy Data Bank under the accession numbers EMD-8360 and EMD-8396, respectively.

Replicative Fitness. The replicative fitness of the different viral populations was investigated by comparing their growth curves in E. coli host cultures. The growth study was performed in duplicate in host E. coli strain C3000 (ATCC 15597, LGC Standards, France). This strain was used in order to exclude that the populations only evolved to adapt to the host strain used during the experimental evolution. To conduct the growth experiment, MS2 was added to the host in exponential growth phase at a targeted MOI of 0.01. A low MOI was chosen in order to ensure an excess amount of host cells such that competition among phages for hosts was minimized. The infected culture was incubated during 4-5 h. At intervals of $1 \mathrm{~h}$, sample aliquots were taken, and infective virus titers were enumerated immediately. Additionally, the viral RNA was extracted, and the number of genome copies was measured by qRT-PCR.

In order to quantitatively compare the one-step growth of the populations of interest, the phage growth curve was fitted to the Modified Gompertz model. This model describes the MS2 growth as a succession of lag (eclipse), exponential growth, and asymptote $^{20,21}$

$$
\log \left(\frac{N}{N_{0}}\right)=A \cdot \exp \left\{-\exp \left[\frac{\mu e}{A}(\lambda-t)+1\right]\right\}
$$

where $A$ represents the asymptote of the growth curve $(\log (N /$ $\left.N_{0}\right)$ ) where $N$ is the concentration of infective viruses or genome copies); $\mu$ is the specific growth rate at log phase (hour ${ }^{-1}$ ); and $\lambda$ is the lag period between infection initiation and the time point when phage progeny begin to appear (hours). Model coefficients for the Modified Gompertz model were obtained in GraphPad Prism by minimizing the sum of squares of the difference between the observed and modeled data for each experiment separately. The relative replicative fitness of the different populations was compared by the selection coefficient $s^{22}$

$$
s=1-\mu / \mu_{-\mathrm{WT}}
$$

where $\mu$ and $\mu_{\text {wT }}$ are the log phase growth rates of the evolved populations and wild-type, respectively. The evolved population has a superior replicative fitness if $s<0$.

Inactivation Experiments with Other Inactivating Agents. Inactivation experiments with free chlorine, $\mathrm{UV}_{254}$, and heat were conducted, and the associated inactivation rate constants $k_{\mathrm{FC}}, k_{\mathrm{UV}}$, and $k_{\text {heat }}$ were determined as described in the Supporting Information.

Statistical Analysis. To determine if the inactivation kinetics or growth rates significantly differed between two or more viral populations, the paired $t$ test analysis or regular oneway ANOVA was applied, respectively, whereby the threshold $p$-value for statistical significance was 0.05 . The goodness-of-fit of the data was evaluated based on the coefficient of determination $\left(R^{2}\right)$, which was determined by GraphPad Prism.

\section{RESULTS AND DISCUSSION}

Emergence of $\mathrm{ClO}_{2}$-Resistant MS2. In order to promote the emergence of $\mathrm{ClO}_{2}$-resistant populations, we subjected five replicates of the same starting (wild-type) population to $\mathrm{ClO}_{2}$ exposure. Of these, two populations (E01 and E02) exhibited resistance to $\mathrm{ClO}_{2}$ after 30 and 22 inactivation-regrowth cycles, respectively. Resistance was manifested in significantly slower inactivation kinetics compared to the wild-type population (Figure 1). Interestingly, as further discussed below, resistance also developed in three of the five nonexposed controls that underwent dilution-regrowth cycles and were thus not exposed to $\mathrm{ClO}_{2}$ (populations NE01, NE02, and NE03).

As expected from previous studies, ${ }^{23} \mathrm{ClO}_{2}$ inactivation of MS2 deviated from first-order Chick-Watson kinetics (Figure 1A) but could be well-described by the Hom model (eq 1). For each individual inactivation experiment, the rate constant $k_{\mathrm{ClO}_{2}}$ and parameters $m$ and $n$ were determined. The $m$ and $n$ values differed by less than $8 \%$ and $23 \%$ across all experiments, respectively, and variations in $m$ and $n$ over this range changed the associated values of $k_{\mathrm{ClO}_{2}}$ by less than $4 \%$. Consequently, $m$ and $n$ were treated as constant parameters for this study. Their values were determined by simultaneously fitting the Hom model to all the experiments conducted and correspond to 0.396 and 0.756 , respectively. The only variable in the Hom model was thus $k_{\mathrm{ClO}_{2}}$.

Among the five resistant populations, E01 and NE01 demonstrated the highest extent of resistance, as quantified by the relative decrease in $k_{\mathrm{ClO}_{2}}$ compared to $k_{\mathrm{ClO}_{2}}$ of the WT population $\left(\frac{k_{\mathrm{ClO}_{2}}}{k_{\mathrm{ClO}_{2}-} \mathrm{WT}} ;\right.$ Figure $\left.1 \mathrm{~B}\right)$. While the titer of the WT 


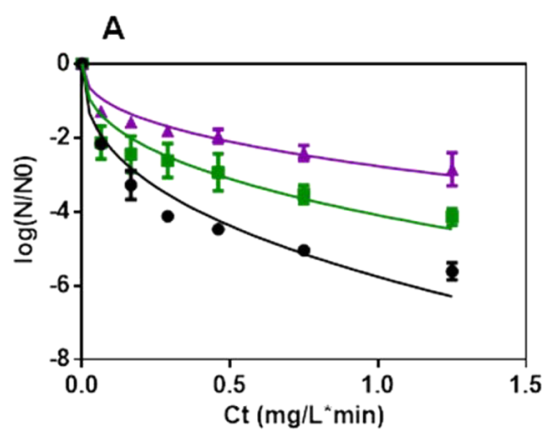

B

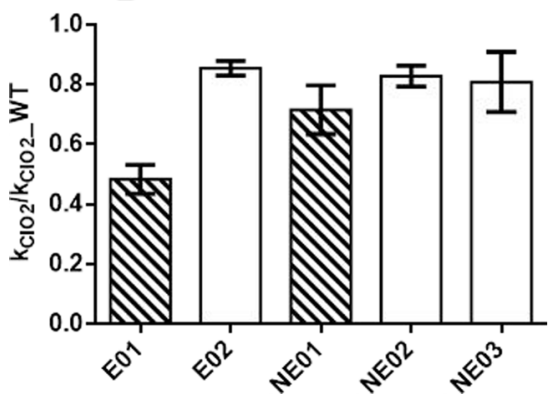

Figure 1. (A) Disinfection kinetics of MS2 upon treatment by $\mathrm{ClO}_{2}$. The $\mathrm{ClO}_{2}$ concentration was maintained at $0.5 \mathrm{mg} / \mathrm{L}$. Black circles: wild-type (WT) population; purple triangles: $\mathrm{ClO}_{2}$ exposed population (E01); green squares: nonexposed population (NE01). The corresponding fits to the Hom model are shown as solid lines. Error bars represent the range of two replicate experiments. (B) The $\frac{k_{\mathrm{ClO}_{2}}}{k_{\mathrm{ClO}_{2}-} \mathrm{WT}}$ of the resistant MS2 populations (two exposed populations E01-02 and three nonexposed populations NE01-03). The $\mathrm{ClO}_{2}$ concentration was maintained at $0.5 \mathrm{mg} / \mathrm{L}$ throughout each experiment. The error bars represent the propagated standard deviation of triplicate experiments. $t$ test showed significant differences in the $k_{\mathrm{ClO}_{2}}$ values between WT and the evolved populations at the 95\% confidence level: E01 $(p<0.0001)$, E02 (0.01), NE01 (0.002), NE02 (0.007), and NE03 (0.02). Striped bars indicate the two samples that were fully characterized phenotypically, while the other evolved populations were subjected to genome sequencing only.

population decreased by $6 \operatorname{logs}$ at a $\mathrm{Ct}$ value of $1 \mathrm{mg} / \mathrm{L} * \min$ upon exposure to $0.5 \mathrm{mg} / \mathrm{L} \mathrm{ClO}_{2}$, the infective E01 and NE01 concentration was reduced by less than 3 logs and 4 logs, respectively (Figure 1A). This resistance persisted at higher $\mathrm{ClO}_{2}$ concentrations: when raising the $\mathrm{ClO}_{2}$ concentration from 0.5 to $2 \mathrm{mg} / \mathrm{L}$, both E01 and NE01 populations remained more resistant than the wild-type (Figure S2, SI).

While $\mathrm{ClO}_{2}$-exposed populations (E populations) developed resistance toward the disinfectant after repeated challenges, the nonexposed samples (NE populations) also became resistant to a similar extent (Figure 1B). From this observation we conclude that $\mathrm{ClO}_{2}$ exposure was not necessary for the emergence of resistance. Instead, a common pressure present in both exposed and nonexposed samples must have played the dominant role in promoting the emergence of the resistance. Both the exposed and nonexposed virus populations encountered the pressure to efficiently and repeatedly expand the population number under strong bottleneck events. The enhanced ability to proliferate may also benefit the survival under disinfection by $\mathrm{ClO}_{2}$. For example, a mutant with a particularly efficient host binding mechanism could both replicate more efficiently and thus dominate regrowth under bottleneck events, as well as better counteract the effects of
$\mathrm{ClO}_{2}$ on the host binding mechanism. Thus, the effect of $\mathrm{ClO}_{2}$ exposure as a stressor in the emergence of resistance was seemingly masked by the genetic drift resulting from the frequent regrowth from low virus numbers. A similar conclusion was obtained by Sheldon et al., who reported that the passage of hepatitis $C$ virus in cell culture resulted in viral resistance to drugs. ${ }^{24}$ It was suggested that the cause of drug resistance was the increased replicative fitness acquired during passage. An alternative explanation for the resistance observed in the nonexposed populations is contamination by exposed viruses. However, the fact that the $\mathrm{E}$ and $\mathrm{NE}$ populations are not genetically identical (see below) indicates that contamination did not occur.

Genotypic Characterization of $\mathrm{ClO}_{2}$ Resistant MS2. The MS2 genome is composed of 3569 nucleotides and encodes for an assembly protein (A protein), a coat protein, a lysis protein, and a replicase. To explore the genetic basis of resistance, the genomes of the WT and the five resistant populations were sequenced. In addition, two evolved populations (E03 and E04) that did not show a significant resistance were included in the analysis. Among these populations, a total of 16 different nucleotide substitutions were identified in the consensus sequences (Table 1). However, the fixation of mutations was only partly reproducible among the different resistant populations, suggesting different resistance pathways. Additionally, the mutation spectrum between exposed and nonexposed resistant populations varied considerably. The exposed populations accumulated more mutations in the A protein (seven, of which five nonsynonymous) than the nonexposed ones (three, all nonsynonymous), while nonexposed populations possessed more synonymous mutations in the replicase-encoding region (four versus two). Of the mutations incurred in the four exposed populations, seven were also found in one of the nonexposed populations. However, the populations containing the same mutations additionally exhibited divergent ones. These findings, while complex, are consistent with previous literature reports on the genomic content of resistant mutants. In the viral adaptation study of Wichman et al., ${ }^{25}$ it was revealed that different amino acid substitutions were identified among experimental replicates, and thus they suggested different trajectories during the adaptation of bacteriophage $\phi \mathrm{X} 174$ to high temperature and a novel host. It was also demonstrated that more than one nucleotide substitution can emerge in influenza A virus populations causing resistance to oseltami$\operatorname{vir}^{26,27}$ and that therefore biological replicates become equally resistant by the beneficial effect caused by different mutations. $^{26,28}$ Finally, Borderia et al. showed that the adaptation of influenza $A$ virus to a new environment and selection pressure not only occurred by single dominant mutations but also depended on the presence of secondary mutations (minority variants) on the population level. The group contribution of these minority variants could counteract the beneficial effect of the main mutation and induce a fitness decrease compared to the mutation alone. ${ }^{28}$ Overall, resistance development can thus be a more diverse and complex process than the fixation of a single mutation with the largest beneficial effect in the population.

Table 1 also shows the ranking of the extent of resistance exhibited in the sequenced populations, where 1 indicates the most (E01) and 4 the nonresistant populations (E03 and E04). E01 was more resistant than NE01 by possessing two additional mutations in A protein (Y102F and I238V). This suggests that 
Table 1. Mutations Identified in the Virus Populations with Reference to the Genome Sequence of WT ${ }^{b}$

\begin{tabular}{|c|c|c|c|c|c|c|c|c|c|}
\hline \multirow[b]{2}{*}{ position } & \multicolumn{2}{|c|}{ mutation } & \multicolumn{7}{|c|}{ population } \\
\hline & NT & AA & E01 & NE01 & NE02 & E02 & NE03 & E03 & E04 \\
\hline \multirow[t]{8}{*}{ A protein } & G155T & R9M & $\sqrt{ }$ & $\sqrt{ }$ & & & & & \\
\hline & $\mathrm{C} 186 \mathrm{~T}$ & - & & & & 0 & & & \\
\hline & $\mathrm{A} 434 \mathrm{~T}$ & $\mathrm{Y} 102 \mathrm{~F}$ & $\sqrt{ }$ & & & & & & \\
\hline & $\mathrm{A} 467 \mathrm{~T}$ & Y113F & $\sqrt{ }$ & $\sqrt{ }$ & & & & & \\
\hline & C513T & - & & & & & & & O \\
\hline & A841G & $\mathrm{I} 238 \mathrm{~V}$ & $\sqrt{ }$ & & & & & & \\
\hline & A1184G & Q352R & & & & & & $\sqrt{ }$ & \\
\hline & C1247A & S373Y & & & $\sqrt{ }$ & & & & \\
\hline \multirow[t]{4}{*}{ coat protein } & $\mathrm{T} 1442 \mathrm{G}$ & - & & & & 0 & & & \\
\hline & A1443G & N36D & $\sqrt{ }$ & $\sqrt{ }$ & & & & & \\
\hline & G1656T & A107S & & & & & $\sqrt{ }$ & $\sqrt{ }$ & $\sqrt{ }$ \\
\hline & $\mathrm{C} 1697 \mathrm{~A}$ & - & & & & 0 & $\bigcirc$ & 0 & 0 \\
\hline lysis protein & $\mathrm{C} 1697 \mathrm{~A}$ & P7Q & & & & $\sqrt{ }$ & $\sqrt{ }$ & $\sqrt{ }$ & $\sqrt{ }$ \\
\hline \multirow[t]{4}{*}{ replicase } & T1946C & - & & & & & 0 & 0 & 0 \\
\hline & $\mathrm{T} 2642 \mathrm{C}$ & - & & & & & 0 & & \\
\hline & $\mathrm{C} 2889 \mathrm{~T}$ & - & & & 0 & & & & \\
\hline & A3239G & - & & & & & 0 & 0 & 0 \\
\hline resistance ranking & & & 1 & 2 & 3 & 3 & 3 & $4^{a}$ & $4^{a}$ \\
\hline
\end{tabular}

${ }^{a}$ Not resistant. ${ }^{b}$ The amino acid position was counted from the initiation of each protein. The ticks and the circles represent nonsynonymous and synonymous mutations, respectively. Nucleotide position 1967 contributes to both the coat protein and the lysis protein region. While it was synonymous in the ORF of coat protein, it caused an amino acid change from proline (P) to glutamine (Q) in the lysis protein.

at least one of these two mutations was directly linked to the increased resistance. NE01, which possessed three mutations, was ranked second in resistance; therefore, at least one of the three mutations exhibited by this population contributed to resistance. Populations E03 and E04, which did not demonstrate significant resistance, nevertheless exhibited six mutations each. Of these, five were shared and were also present in the resistant NE03, which had only one additional (synonymous) mutation. Combined, these results indicate that either mutations exist in E03 and E04 (A1184G and synonymous mutation C513T) that compensated for resistance-inducing mutations or that the synonymous mutation in NE03 can contribute to resistance. The potential role of synonymous mutations is supported by findings by Tubiana et al., who suggested that that synonymous mutations in viral RNA can influence the structure of packaged genome in single stranded RNA viruses, and higher RNA compactness facilitates virus maturation and assembly. ${ }^{29}$ Therefore, even though MS2 inactivation by $\mathrm{ClO}_{2}$ does not involve genome damage, synonymous mutations could still influence the virus structure and functions and hence its disinfection susceptibility.

Effect of Mutations on Virus Structure. The majority of the nonsynonymous mutations were located in the structural proteins (A protein and coat protein; Table S1, SI). The only copy of A protein is located near the 5-fold axis of the protein capsid and plays several roles in MS2 life cycle. First, the A protein helps the virus to bind to the E. coli F-pilus. Once bound, the retraction of F-pilus pulls the A protein from the capsid leaving a pore such that the viral genome can exit the protein capsid and enter the cell as an A protein-RNA complex. ${ }^{30}$ The A protein furthermore promotes the virion assembly process by circularizing the viral RNA. ${ }^{30-32}$ The mutations in the A protein may thus influence the binding to the host cells, genome injection, and virus assembly. The current knowledge of A protein function is still insufficiently detailed to allow us to pin down the significance of each mutation with respect to their influence on viral functions. However, cryo-EM reconstruction of the wild-type and resistant viral particles (NE01) revealed that the biggest structural differences lie in the A protein; the corresponding cryo-EM densities show a Pearson correlation coefficient of 0.84 (Figure 2). Specifically, a more protruding tip can be observed in the A protein of NE01 compared to the wild-type (Figure 2A and $\mathrm{C}$ ). This result supports our hypothesis that resistance arises from changes in virus motifs associated with host binding.

The MS2 coat proteins serve as the main scaffold of the virus particle, and it encapsulates and protects the viral genome. Upon virion maturation, RNA binds to coat protein dimers and consequently triggers the assembly of the capsid. ${ }^{31}$ Mutations in the coat protein may thus influence virion assembly and structure. Figure S3A (SI) illustrates an MS2 coat protein monomer containing the observed nonsynonymous mutations along with some other interesting residues denoted. Mutation $\mathrm{N} 36 \mathrm{D}$, which was the only mutation present in the coat protein of the two most resistant populations (E01 and NE01; Table 1 ), is located on the DE loop. While not involved in RNA binding (shown in green in Figure S3A, SI), the DE loop is implicated in the interaction between coat protein dimers to form the full MS2 capsid (Figure S3B and Figure S3C, SI). ${ }^{33}$ The substitution of a polar, uncharged asparagine at position 36 by a negatively charged aspartic acid causes a change in the local charge distribution, and this, in turn, could influence the overall protein configuration. Correspondingly, cryo-EM reconstruction revealed a small but measurable difference in the structure of the protein capsid compared to the wild-type (Pearson correlation coefficient of 0.94; Figure 2A), which may influence binding efficiency and specificity. 

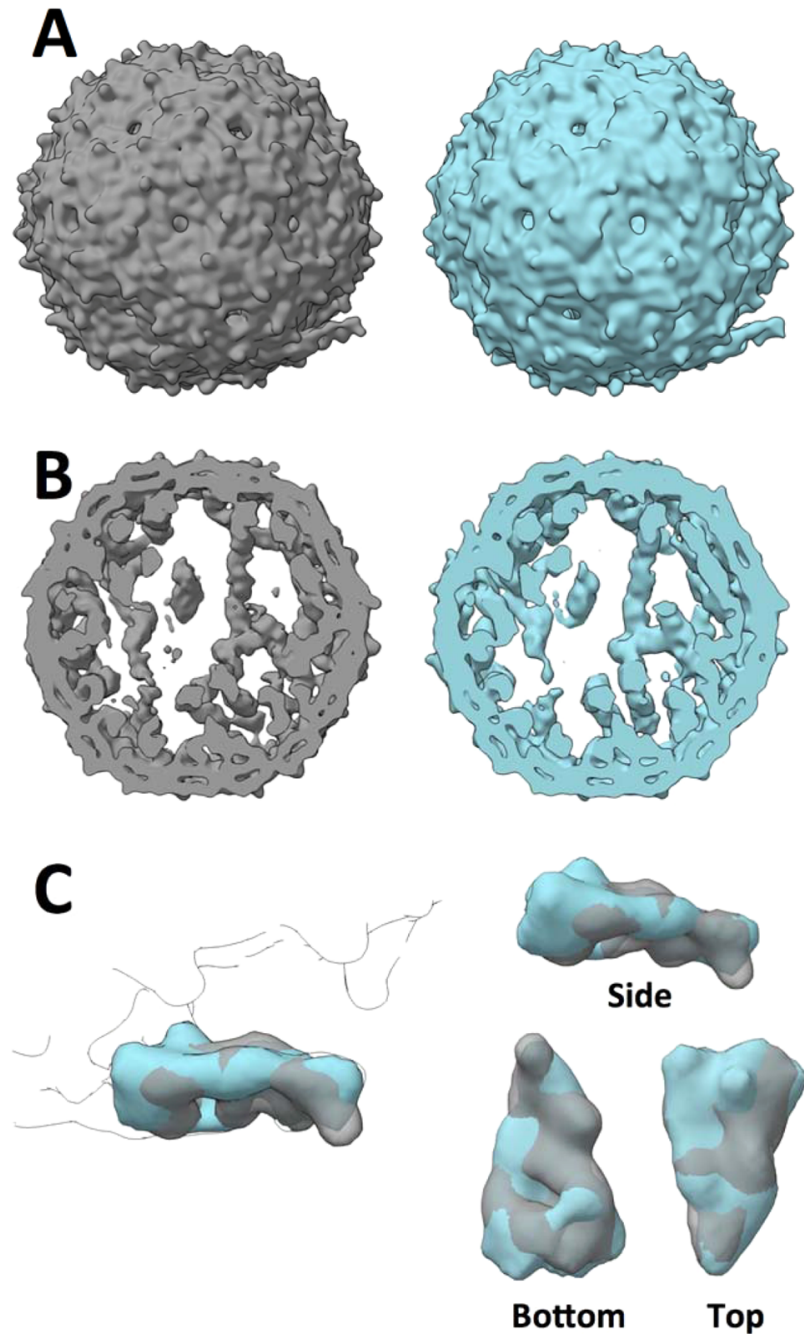

Figure 2. Cryo-EM 3D reconstruction of NE01 (gray) and WT (turquoise) virion particles. (A) The whole virions; (B) Virion particles sliced open exposing RNA organized in single- and doublestranded segments; (C) Superposition of A protein of WT and NE01. The Pearson correlation coefficients for whole virion, coat protein capsid, RNA, and A protein are 0.94, 0.92, 0.89, and 0.84, respectively.

Finally, differences were also found between the RNA structures of the wild-type and NE01 (Pearson correlation coefficient $=0.92$; Figure $2 \mathrm{~B}$ ). These differences may influence RNA-coat protein interaction and thus play a part in packaging and assembly efficiency. The RNA structure modification may furthermore influence the stability of the genome and the whole virion.

Resistance Mechanism. To understand how the genetic and structural changes discussed above cause resistance, they must be linked to phenotypic effects. We therefore investigated the most resistant populations with respect to two phenotypic traits that may be linked to resistance, namely the ability to attach to the host cell (E01 only) and the tendency to form aggregates as protection against disinfection (E01 and NE01).

Host Binding. As stated in the Introduction, we hypothesize that resistance of MS2 to $\mathrm{ClO}_{2}$ is linked to the virus' sustained ability to attach to its host after $\mathrm{ClO}_{2}$ exposure. To test this hypothesis, the extent of host binding of the wild-type and the resistant populations E01 and NE01 were measured as a function of $\mathrm{ClO}_{2}$ dose. As shown in Figure 3, the loss in host binding with increasing $\mathrm{ClO}_{2}$ exposure was indeed drastically less pronounced for E01 and NE01 compared to WT.

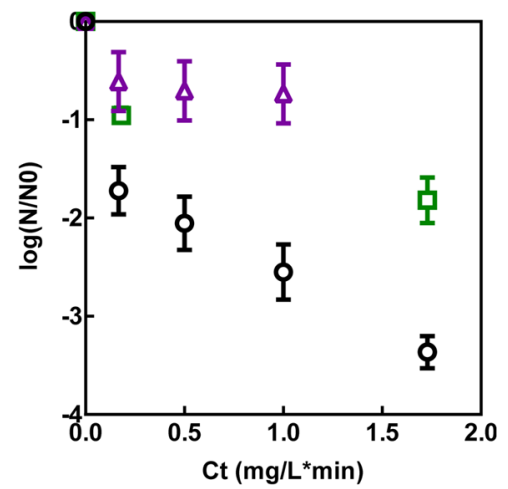

Figure 3. Decrease in host binding as a function of $\mathrm{ClO}_{2}$ dose for WT, E01, and NE01. Black circles: wild-type population; purple triangles: E01 population; green squares: NE01 population. The error bars represent the standard deviation of triplicate samples.

The more stable host binding of the resistant populations compared to WT is consistent with the differences in genome composition and morphology discussed above. The 3D reconstruction highlighted that the most important structural changes occurred in the A protein, which plays a vital role in the binding of MS2 to its host. ${ }^{31}$ This is consistent with the majority of nonsynonymous mutations in E01 and NE01 being found in the A protein (Table 1). Two of these mutations affected the chemical reactivity of the A protein toward $\mathrm{ClO}_{2}$. Specifically, it is well-known that $\mathrm{ClO}_{2}$ preferentially reacts with a subset of amino acids, namely cysteine, tyrosine, tryptophan, histidine, and proline. ${ }^{34}$ Among the mutations identified on the A protein, two mutations converted $\mathrm{ClO}_{2}$-reactive tyrosine (Y102 and Y113) to nonreactive phenylalanine. Interestingly, these two tyrosine residues are located in the region of the $\mathrm{A}$ protein that is among the most rapidly degraded during exposure to $\mathrm{ClO}_{2}{ }^{19}{ }^{19}$ We thus propose that the substitutions to nonreactive residues enhance the chemical integrity of the $\mathrm{A}$ protein upon exposure to $\mathrm{ClO}_{2}$. In addition, the elongation of the A protein structure (Figure 2) may confer physical advantages to binding. Overall, these effects hence allow the resistant populations to better maintain their ability to attach to the host compared to the WT population.

Aggregation. It is known that surface charge modification can lead to a change in the aggregation status of viral particles, ${ }^{35}$ which in turn can influence the susceptibility of the population toward disinfectants. ${ }^{36}$ Of the mutations found in E01 and NE01, only one (N36D) leads to a change in charge of the coat protein. This change, however, did not result in enhanced aggregation (see the SI for details). Aggregation could thus be excluded as a cause for enhanced resistance.

Are Resistant Viruses Likely To Proliferate? A fundamental assumption in evolution is the existence of trade-offs; ${ }^{37,38}$ the ability to survive under a given stressor is thus expected to come at the expense of another phenotypic trait. The extent and nature of the specific trade-offs contribute to the likelihood of a mutant to thrive in a given environment. To assess if disinfection-resistant viruses are likely to proliferate, they must be compared with the wild-type with respect to their ability to produce infective progeny and to withstand other stressors, including those typically applied to control viruses. We thus assessed the replicative fitness of resistant populations 
E01 and NE01 compared to WT and quantified their crossresistance to a suite of other inactivating agents.

Replicative Fitness. The replicative fitness, which informs about the virus growth kinetics and final population size, was determined for resistant and wild-type populations in an E. coli host (C3000). Growth curves were established for WT, E01, and NE01, to characterize the kinetics of production of both infective viruses and total genome copies (Figure S4, SI). The growth curves were fitted to the Modified Gompertz eq (eq 2). Table S2 (SI) summarized the associated model parameters lag phase, growth rate, and final concentration. The replicative fitness of the different populations was compared by the selection coefficient $s$ for the infective populations (eq 3). For E01 and NE01, positive $s$ values were obtained (0.15 and 0.16), indicating a slightly reduced fitness compared to the WT ( $p=$ 0.04 and 0.02 ).

The kinetics of genome replication (Figure S4B, SI) revealed further differences between the populations tested. Specifically, NE01 showed a significantly shorter lag period than WT (by $73 \%, p<0.0001$ ) and higher asymptote (by $32 \%, p<0.0001$ ). Despite this greater production of total genome copies compared to the other populations, the output of infectious virus was not higher for NE01. We thus conclude that NE01 had a propensity to produce more defective viral particles during infection.

Cross-Resistance to Other Inactivating Agents. If virus resistance to one disinfectant causes cross-resistance to other disinfectants, their inactivation would become problematic and their proliferation in the environment may pose a serious concern. To assess the cross-resistance of E01 and NE01 to other inactivating treatments, we compared the inactivation kinetics of our resistant and wild-type populations by $\mathrm{UV}_{254}$, FC, and heat (Figure 4 and Table S3, SI). While $\mathrm{ClO}_{2}$ only damages MS2 proteins, $\mathrm{UV}_{254}$ mainly targets the genome, where it promotes the formation of pyrimidine dimers and thereby inhibits genome replication. FC causes multiple alterations to both genome and proteins, including protein oxidation, chlorination, carbonylation, and backbone cleavage. Finally, heat causes the denaturation of protein capsid and thus disrupts specific structures needed for host cell binding without inducing chemical modifications. ${ }^{19}$ Given the diverse mode of action of these inactivating agents, it is interesting to assess if the resistance observed herein is specific to $\mathrm{ClO}_{2}$, or if it is also manifested toward other inactivation agents acting by different mechanisms.

For each of the three inactivation agents tested, the inactivation kinetics of the WT and the $\mathrm{ClO}_{2}$-resistant populations were mostly similar (Figure 4), indicating that $\mathrm{ClO}_{2}$-resistant viruses remain susceptible to other inactivating treatments. For $\mathrm{UV}_{254}$ treatment, the inactivation rate constants of the different virus populations were not significantly different (Table S3, SI). This result was expected, given that UV inhibits genome replication, whereas MS2 resistance to $\mathrm{ClO}_{2}$ is rooted in the protein-mediated binding function. In contrast, the $\mathrm{ClO}_{2}$ resistant populations also exhibited a small extent of resistance toward FC, albeit only at a $90 \%$ confidence level (Table S3, SI). This may indicate that the enhanced protein stability of the mutants toward $\mathrm{ClO}_{2}$ has a similar effect on FC. This effect is attenuated, however, because $\mathrm{FC}$ is less selective compared to $\mathrm{ClO}_{2}$ in the targeted protein residues, and because inactivation by $\mathrm{FC}$ involves a significant genome component. ${ }^{19}$

Finally, inactivation by heat at $72{ }^{\circ} \mathrm{C}$ was significantly faster for E01 than for WT, while the difference between NE01 and
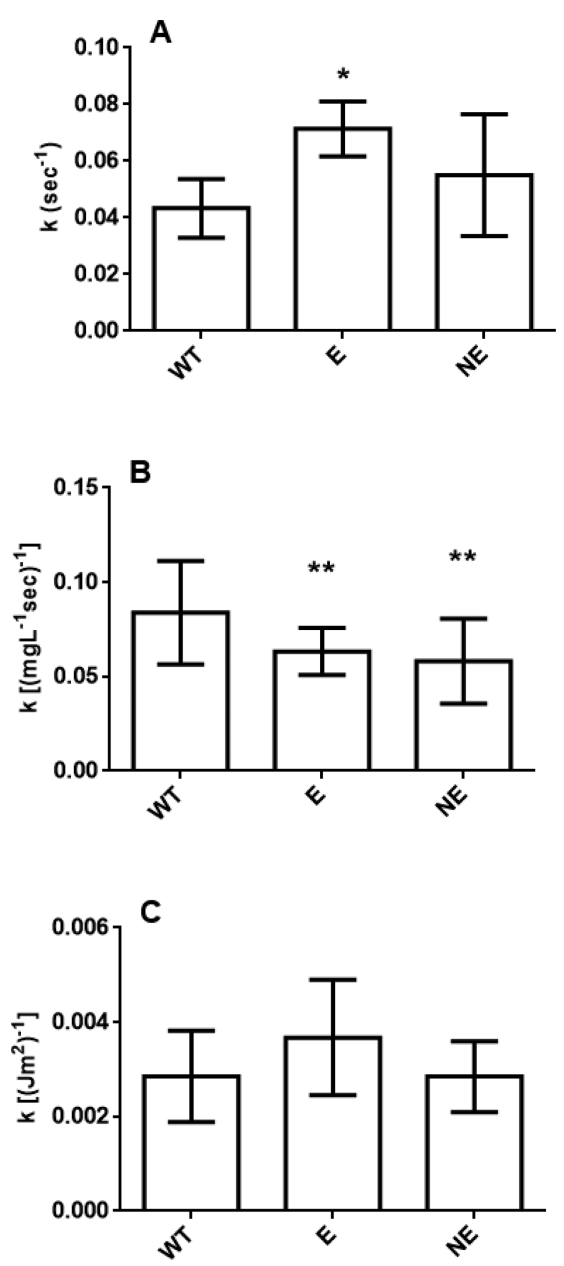

Figure 4. Inactivation rate constants of WT, E01, and NE01 for treatment by heat (A), free chlorine (B), and $\mathrm{UV}_{254}(\mathrm{C})$. Rate constants were determined from simultaneous fits of the data to equations S1-S3 (Supporting Information) to duplicate experiments. The error bars represent the associated $95 \%$ confidence intervals. Statistically significant differences between rate constants of the evolved populations and the WT are indicated by a single asterisk (*) for the $95 \%$ confidence level $(p<0.05)$ and by a double asterisk $(* *)$ for the $90 \%$ confidence level $(p<0.1)$.

WT was not statistically significant. The more $\mathrm{ClO}_{2}$-resistant populations E01 and NE01 were thus the least persistent when challenged by heat. Although heat also acts on proteins, the mode of action does not involve oxidation. Hence the oxidation-resistant mutations did not necessarily contribute to the defense against thermal denaturation. Instead, the mutations decreased the thermal stability of the virus particles and made them less thermotolerant.

Given their only slightly impaired replicative fitness, and their susceptibility to inactivating agents that act by a different mechanism than $\mathrm{ClO}_{2}$, we conclude that $\mathrm{ClO}_{2}$-resistant viruses may be able to circulate but that they can be controlled by other common water and wastewater disinfection methods.

Implications for Virus Control. This study provides evidence for the development of resistance of a bacteriophage to $\mathrm{ClO}_{2}$ disinfection. Resistance arose from different mutations and different mechanisms and could emerge simply through repeated regrowth cycles, without the need for $\mathrm{ClO}_{2}$ exposure. Hence, the mere cycling of a human virus in a population, which involves sequential rounds of excretion and reinfection of 
new hosts, may render a virus population increasingly $\mathrm{ClO}_{2}$ resistant. Furthermore, if the exposure to a disinfectant does accelerate the accumulation of resistance-inducing mutations, then resistant viruses would be most abundant where disinfection is widely used. While these notions remain to be tested, they are supported by the findings that environmental isolates of human viruses can be more resistant to disinfection than the corresponding lab strains ${ }^{13}$ and that some of the resistant strains were isolated from disinfected waters. ${ }^{13,39}$

Many disinfection systems consist of two sequential stages of disinfection, which sometimes involve different disinfectants. ${ }^{40}$ This approach could be beneficial for the inactivation of resistant viruses, since our work shows little evidence of crossresistance, in particular if the two disinfectants act by different mechanisms. However, such a setup may also lead to the selection of mutants that exhibit multiple resistances to different disinfectants. Further work is required to determine if such viruses can emerge, if they can be found in the environment, and if they are likely to proliferate.

Finally, it is known that drug-resistant viruses can circulate for years even after the drug pressure was removed. ${ }^{41,9,10}$ Disinfectant-resistant viruses circulating in the environment could thus potentially preserve their resistance over long periods of time. This work contributes to a better understanding of the pressures that promote such resistant viruses and the possibilities to control them.

\section{ASSOCIATED CONTENT}

\section{S Supporting Information}

The Supporting Information is available free of charge on the ACS Publications website at DOI: 10.1021/acs.est.6b04170.

Additional experimental information on Sanger sequencing, virus aggregation, Cryo-EM analysis and inactivation by $\mathrm{FC}, \mathrm{UV}_{254}$, and heat; diagram of experimental evolution setup; the inactivation kinetics of MS2 with different initial $\mathrm{ClO}_{2}$ concentrations; figures showing MS2 coat protein monomer and dimer interactions at quasi 5-fold and 6-fold axes; one-step growth curve comparison of WT and the mutants and the model fitting parameters; inactivation rate constants in the inactivation experiments using $\mathrm{FC}, \mathrm{UV}_{254}$, and heat (PDF)

\section{AUTHOR INFORMATION}

\section{Corresponding Author}

*Phone: +41 21693 0891. E-mail: tamar.kohn@epfl.ch.

\section{Present Addresses}

${ }^{\S}$ Biozentrum, University of Basel, Klingelbergstrasse 70, 4056 Basel, Switzerland.

"FEI Company, 5350 NE Dawson Creek Drive, Hillsboro, OR 97124, USA.

${ }^{\perp}$ University of Texas Medical Branch, 301 University Blvd, Galveston, TX 77555, USA.

\section{Notes}

The authors declare no competing financial interest.

\section{ACKNOWLEDGMENTS}

This work was funded by the Swiss National Foundation (project number 31003A_138319). We thank Martin Ackermann, Thérèse Sigstam, Lö̈ Decrey, and Michael Mattle for advice with the experimental design and protocols. We acknowledge Pierre Rossi for his laboratory assistance.

\section{REFERENCES}

(1) Aronino, R.; Dlugy, C.; Arkhangelsky, E.; Shandalov, S.; Oron, G.; Brenner, A.; Gitis, V. Removal of viruses from surface water and secondary effluents by sand filtration. Water Res. 2009, 43 (1), 87-96.

(2) Mamane, H.; Shemer, H.; Linden, K. G. Inactivation of E. coli, B. subtilis spores, and MS2, T4, and T7 phage using UV/H2O2 advanced oxidation. J. Hazard. Mater. 2007, 146 (3), 479-486.

(3) Bryant, E. A.; Fulton, G. P.; Budd, G. C. Disinfection alternatives for safe drinking water; Van Nostrand Reinhold: New York, 2003.

(4) Duffy, S.; Shackelton, L. A.; Holmes, E. C. Rates of evolutionary change in viruses: patterns and determinants. Nat. Rev. Genet. 2008, 9 (4), 267-276.

(5) Simmonds, P. Genetic diversity and evolution of hepatitis $C$ virus - 15 years on. J. Gen. Virol. 2004, 85 (11), 3173-3188.

(6) Lauring, A. S.; Frydman, J.; Andino, R. The role of mutational robustness in RNA virus evolution. Nat. Rev. Microbiol. 2013, 11 (5), 327-336.

(7) Domingo, E.; Quer, E. J.; Novella, S.; John, J. Basic concepts in RNA virua evolution. FASEB J. 1996, 10 (8), 859-864.

(8) Sanjuán, R.; Nebot, M. R.; Chirico, N.; Mansky, L. M.; Belshaw, R. Viral mutation rates. J. Virol. 2010, 84 (19), 9733-9748.

(9) Hué, S.; Gifford, R. J.; Dunn, D.; Fernhill, E.; Pillay, D. Demonstration of sustained drug-resistant human immunodeficiency virus type 1 lineages circulating among treatment-näive individuals. $J$. Virol. 2009, 83 (6), 2645-2654.

(10) Mbisa, J. L.; Fearnhill, E.; Dunn, D. T.; Pillay, D.; Asboe, D.; Cane, P. A. Evidence of Self-Sustaining Drug Resistant HIV- 1 Lineages Among Untreated Patients in the United Kingdom. Clin. Infect. Dis. 2015, 61 (5), 829-836.

(11) Bates, R. C.; Shaffer, P. T.; Sutherland, S. M. Development of poliovirus having increased resistance to chlorine inactivation. Appl. Environ. Microbiol. 1977, 34 (6), 849-853.

(12) Maillard, J.-Y.; Hann, A. C.; Perrin, R. Resistance of Pseudomonas aeruginosa PAO1 phage F116 to sodium hypochlorite. J. Appl. Microbiol. 1998, 85 (5), 799-806.

(13) Payment, P.; Tremblay, M.; Trudel, M. Relative resistance to chlorine of poliovirus and coxsackievirus isolates from environmental sources and drinking water. Appl. Environ. Microbiol. 1985, 49 (4), 981-983.

(14) Wigginton, K. R.; Kohn, T. Virus disinfection mechanisms: the role of virus composition, structure, and function. Curr. Opin. Virol. 2012, 2 (1), 84-89.

(15) Pecson, B. M.; Martin, L. V.; Kohn, T. Quantitative PCR for determining the infectivity of bacteriophage MS2 upon inactivation by heat, UV-B radiation, and singlet oxygen: advantages and limitations of an enzymatic treatment to reduce false-positive results. Appl. Environ. Microbiol. 2009, 75 (17), 5544-5554.

(16) Rice, E. W.; Baird, R. B.; Eaton, A. D.; Clesceri, L. S. Standard Methods for the Examination of Water and Wastewater, 22nd ed.; American Public Health Association, American Water Works Association, Water Environment Federation: Washington, DC, 2012.

(17) Hoigne, J.; Bader, H. Kinetics of Reactions of Chlorine Dioxide (OC10) in water-I. Rate constants for inorganic and organic compounds. Water Res. 1994, 28 (1), 45-55.

(18) Haas, C. N.; Joffe, J. Disinfection under dynamic conditions: modification of Hom's model for decay. Environ. Sci. Technol. 1994, 28 (7), 1367-1369.

(19) Wigginton, K. R.; Pecson, B. M.; Sigstam, T.; Bosshard, F.; Kohn, T. Virus inactivation mechanisms: impact of disinfectants on virus function and structural integrity. Environ. Sci. Technol. 2012, 46 (21), 12069-12078.

(20) Zwietering, M. H.; Jongenburger, I.; Rombouts, F. M.; van 't Riet, K. Modeling of the bacterial growth curve. Appl. Environ. Microbiol. 1990, 56 (6), 1875-1881.

(21) Kim, Y. W.; Lee, S. H.; Hwang, I. G.; Yoon, K. S. Effect of temperature on growth of vibrio paraphemolyticus and Vibrio vulnificus in flounder, salmon sashimi and oyster meat. Int. J. Environ. Res. Public Health 2012, 9, 4662-4675. 
(22) Bonhoeffer, S.; Barbour, A. D.; De Boer, R. J. Procedures for reliable estimation of viral fitness from time-series data. Proc. $R$. Soc. London, Ser. B 2002, 269 (1503), 1887-1893.

(23) Sigstam, T.; Rohatschek, A.; Zhong, Q.; Brennecke, M.; Kohn, $\mathrm{T}$. On the cause of the tailing phenomenon during virus disinfection by chlorine dioxide. Water Res. 2014, 48 (1), 82-89.

(24) Sheldon, J.; Beach, N. M.; Moreno, E.; Gallego, I.; Piñeiro, D.; Martínez-Salas, E.; Gregori, J.; Quer, J.; Esteban, J. I.; Rice, C. M.; et al. Increased replicative fitness can lead to decreased drug sensitivity of hepatitis C virus. J. Virol. 2014, 88 (20), 12098-12111.

(25) Wichman, H. A.; Badgett, M. R.; Scott, L. A.; Boulianne, C. M.; Bull, J. J. Different Trajectories of Parallel Evolution During Viral Adaptation. Science (Washington, DC, U. S.) 1999, 285 (5426), 422424.

(26) Foll, M.; Poh, Y. P.; Renzette, N.; Ferrer-Admetlla, A.; Bank, C.; Shim, H.; Malaspinas, A. S.; Ewing, G.; Liu, P.; Wegmann, D.; et al. Influenza Virus Drug Resistance: A Time-Sampled Population Genetics Perspective. PLoS Genet. 2014, 10 (2), e1004185.

(27) Renzette, N.; Caffrey, D. R.; Zeldovich, K. B.; Liu, P.; Gallagher, G. R.; Aiello, D.; Porter, A. J.; Kurt-Jones, E. A.; Bolon, D. N.; Poh, Y.P.; et al. Evolution of the influenza A virus genome during development of oseltamivir resistance in vitro. J. Virol. 2014, 88 (1), $272-281$.

(28) Bordería, A. V.; Isakov, O.; Moratorio, G.; Henningsson, R.; Agüera-González, S.; Organtini, L.; Gnädig, N. F.; Blanc, H.; Alcover, A.; Hafenstein, S.; et al. Group Selection and Contribution of Minority Variants during Virus Adaptation Determines Virus Fitness and Phenotype. PLoS Pathog. 2015, 11 (5), e1004838.

(29) Tubiana, L.; Božič, A. L.; Micheletti, C.; Podgornik, R. Synonymous mutations reduce genome compactness in icosahedral ssRNA viruses. Biophys. J. 2015, 108 (1), 194-202.

(30) Dent, K. C.; Thompson, R.; Barker, A. M.; Hiscox, J. A.; Barr, J. N.; Stockley, P. G.; Ranson, N. A. The asymmetric structure of an icosahedral virus bound to its receptor suggests a mechanism for genome release. Structure 2013, 21 (7), 1225-1234.

(31) Toropova, K.; Basnak, G.; Twarock, R.; Stockley, P. G.; Ranson, N. A. The three-dimensional structure of genomic RNA in bacteriophage MS2: implications for assembly. J. Mol. Biol. 2008, 375 (3), 824-836.

(32) Olsthoorn, R; van Duin, J. Bacteriophages with ssRNA. eLS; 2011 (accessed Jan 1, 2011).

(33) Stonehouse, N. J.; Valegård, K.; Golmohammadi, R.; van den Worm, S.; Walton, C.; Stockley, P. G.; Liljas, L. Crystal structures of MS2 capsids with mutations in the subunit FG loop. J. Mol. Biol. 1996, 256 (2), 330-339.

(34) Sharma, V. K.; Sohn, M. Reactivity of chlorine dioxide with amino acids, peptides, and proteins. Environ. Chem. Lett. 2012, 10 (3), $255-264$.

(35) Maillard, J.-Y.; Sattar, S. A.; Pinto, F. Virucidal Activity of Microbicides. In Russell, Hugo \& Ayliffe's: Principles and Practice of Disinfection, Preservation and Sterilization; Fraise, A. P., Maillard, J.-Y., Sattar, S. A., Eds.; Blackwell Publishing Ltd: Oxford, 2013; pp 178207.

(36) Mattle, M. J.; Crouzy, B.; Brennecke, M. R.; Wigginton, K.; Perona, P.; Kohn, T. Impact of virus aggregation on inactivation by peracetic acid and implications for other disinfectants. Environ. Sci. Technol. 2011, 45 (18), 7710-7717.

(37) De Paepe, M.; Taddei, F. Viruses' life history: Towards a mechanistic basis of a trade-off between survival and reproduction among phages. PLoS Biol. 2006, 4 (7), 1248-1256.

(38) Goldhill, D. H.; Turner, P. E. The evolution of life history tradeoffs in viruses. Curr. Opin. Virol. 2014, 8, 79-84.

(39) Shaffer, P. T.; Metcalf, T. G.; Sproul, O. J. Chlorine resistance of poliovirus isolants recovered from drinking water. Appl. Environ. Microbiol. 1980, 40 (6), 1115-1121.

(40) U.S. EPA. 2006 Community Water System Survey Volume I: Overview; EPA-815-R-09-001; U.S. Environ. Prot. Agency: 2006.

(41) Gillman, A.; Muradrasoli, S.; Söderström, H.; Holmberg, F.; Latorre-Margalef, N.; Tolf, C.; Waldenström, J.; Gunnarsson, G.;
Olsen, B.; Järhult, J. D. Oseltamivir-Resistant Influenza A (H1N1) Virus Strain with an H274Y Mutation in Neuraminidase Persists without Drug Pressure in Infected Mallards. Appl. Environ. Microbiol. 2015, 81 (7), 2378-2383. 